\title{
Creative Strategies Implemented During the Coronavirus Pandemic That Will Impact the Future of Cancer Care
}

\author{
Jessica Sugalski, MPPA ${ }^{1}$; Theresa Franco, MSN, RN²; Lawrence N. Shulman, MD³; Pelin Cinar, MD, MS; \\ James Bachman, MPA ${ }^{5}$; Jennie R. Crews, MD, MMM6; MiKaela Olsen, DNP, APRN, AOCNS7; \\ Alyssa Schatz, MSW ${ }^{1}$; and Timothy Kubal, MD, MBA ${ }^{8}$
}

\begin{abstract}
The coronavirus pandemic has significantly impacted operations at leading cancer centers across the United States. In the midst of the chaos, at least one silver lining has emerged: the development of new, creative strategies for delivering cancer care that are likely to continue post pandemic. The NCCN Best Practices Committee, which is composed of senior physician, nursing, and administrative leaders at NCCN Member Institutions, conducted a webinar series in June 2020 highlighting the most promising and effective strategies to date. Experts from NCCN Member Institutions participated in the series to share their experiences, knowledge, and thoughts about the future of cancer care.
\end{abstract}

J Natl Compr Canc Netw, doi: 10.6004/jnccn.2020.7661 Published online October 30, 2020

\footnotetext{
${ }^{1}$ National Comprehensive Cancer Network, Plymouth Meeting, Pennsylvania; ${ }^{2}$ Fred and Pamela Buffett Cancer Center, Omaha, Nebraska; ${ }^{3}$ Abramson Cancer Center at the University of Pennsylvania, Philadelphia, Pennsylvania; ${ }^{4}$ Department of Medicine, Division of Medical Oncology, University of California San Francisco, San Francisco; ${ }^{5}$ University of Colorado Cancer Center, Aurora, Colorado; ${ }^{6}$ Seattle Cancer Care Alliance, Seattle, Washington; ${ }^{7}$ The Sidney Kimmel Comprehensive Cancer Center at Johns Hopkins, Baltimore, Maryland; and ${ }^{8}$ Moffitt Cancer Center, Tampa, Florida.
}

The coronavirus has been like a tornado crashing through the US health system. Hospitals across the country have been operating in crisis mode since March, postponing nonessential surgeries, delaying treatment, increasing infection control protocols, creating makeshift intensive care unit beds, and deploying unique staffing strategies. This, of course, includes the nation's top comprehensive cancer centers, many of which were already struggling to meet patient demand long before coronavirus made its first appearance. ${ }^{1,2}$

As the country shifts from crisis mode to learning to coexist with coronavirus, some silver linings to the pandemic are beginning to emerge. For centuries we have seen crises push industries to rapidly develop creative strategies and innovations that go on to change the way we do things, even after the crisis is over. Ambulances were invented during the Napoleonic wars of the late 18th century, modern epidemiology was discovered during the 1854 cholera pandemic, and the first plastic surgery techniques emerged during World War I. ${ }^{3}$ What is it about crises that spur innovation? A recent article hypothesized that "crises present us with unique conditions that allow innovators to think and move more freely to create rapid, impactful change." ${ }^{4}$

The NCCN Best Practices Committee decided to examine their pandemic-related innovations more closely and asked NCCN Member Institutions to share creative strategies implemented during the pandemic that have been effective, impactful, and sustainable. The most promising and innovative strategies were featured in a 3-part webinar series that took place in June and included expert speakers from NCCN Member Institutions. This is a summary of the learnings.

\section{Delivering Cancer Care via Telemedicine is Here to Stay}

Although telemedicine in cancer care has existed for years, its adoption has been slow and limited due to barriers related to upfront cost of implementation, licensure, reimbursement, and provider engagement. ${ }^{5,6}$ 
This quickly changed when the pandemic hit the United States in March 2020. Pelin Cinar, MD, MS, Medical Director of Quality and Safety at the UCSF Helen Diller Family Comprehensive Cancer Center, noted that telemedicine visits, which included video and telephone visits, represented approximately $8 \%$ of total oncology visits at her institution in January, but jumped as high as $70 \%$ in April. Similar increases were seen across other NCCN Member Institutions: Seattle Cancer Care Alliance (SCCA) went from $2 \%$ to $38 \%$; University of Colorado Cancer Center went from 0\% to 30\%; and Moffitt Cancer Center went from approximately 10 telemedicine visits per week to $>1,600$ per week at its high point to date. According to the NCCN Best Practices Committee, which conducted weekly calls to discuss cancer center responses to coronavirus in March through June 2020, NCCN Member Institutions conducted $0 \%$ to $10 \%$ of oncology visits via telemedicine before the coronavirus pandemic, and increased these visits to anywhere from $20 \%$ to $80 \%$ as the pandemic moved into their communities. Rates of telemedicine usage often varied in relation to local coronavirus outbreaks.

Telemedicine increases were seen not only in cancer care, but all across the US healthcare system. According to a McKinsey \& Company study, $11 \%$ of Americans used telemedicine in 2019 compared with $46 \%$ in $2020 .^{7}$ It is clear that telemedicine provides a safe way to deliver healthcare during a pandemic. However, there may also be other major benefits to telemedicine expansion that go beyond reducing virus spread.

Lawrence N. Shulman, MD, Deputy Director for Clinical Services at the Abramson Cancer Center at the University of Pennsylvania, discussed the use of telemedicine for palliative care, cancer surveillance, and survivorship. Dr. Shulman noted that, in regard to palliative care, his institution focused on offering telemedicine to the sickest patients whose travel to the cancer center was most difficult or burdensome. Initial data from Abramson Cancer Center indicated that shifting these appointments to telemedicine visits increased consultation rates, decreased no-show rates, increased the number of serious illness care discussions, reduced additional (unnecessary) lines of therapy, and prompted early hospice use. These encouraging results have spurred the cancer center to create a plan to continue providing much of the care for these patients via telemedicine, even after the pandemic has ended.

A large portion of surveillance and survivorship care at Abramson Cancer Center has also shifted to telemedicine during the pandemic. Laboratory tests and imaging are often performed locally prior to the telemedicine visit, and only patients found to require a physical examination or in-person procedure need to travel to the cancer center. Dr. Shulman noted that initial screening for clinical trials is also frequently performed by telemedicine, and anecdotally this has allowed patients from a broader geographic area to access clinical trials. Furthermore, telemedicine monitoring for patients already enrolled on clinical trials has reduced the patient burden of frequent clinic visits at the cancer center.

The University of Colorado Cancer Center also dramatically increased telemedicine visits for supportive care services, according to Jamie Bachman, MPA, Executive Director of Oncology Services. Mr. Bachman discussed the center's ability to quickly pivot from fully in-person services to fully virtual care. A 3-year project was executed in just 3 days when the pandemic landed in Colorado. The entire supportive care team began working from home to create safe physical separation and minimize risk of disease transmission, and converted all social work, nutrition, clinical psychology, and palliative care services to telemedicine visits. This was complemented by virtual offerings for support groups, distress screening, and financial counseling. Patients and providers alike have reported satisfaction with the remote offerings, which will continue (at least partially) at the cancer center for the foreseeable future.

In addition to clearly enriching the care of some patients, the convenience of telemedicine is seen broadly as increasing patient satisfaction. Dr. Cinar provided data from UCSF indicating that patients were just as satisfied with telemedicine visits as they were with in-person visits (January-April 2020). Furthermore, the center experienced an increase in patient satisfaction with access to care for those who had telemedicine visits versus in-person visits. This is not surprising, given that studies conducted before the coronavirus pandemic reported that patients appreciate the convenience of receiving care via telemedicine. ${ }^{8,9}$

The clinical benefits of telemedicine for patients with cancer combined with high patient satisfaction make a strong case for centers to offer an array of telemedicine visits in the future. In fact, when Tim Kubal, MD, MBA, Director of Clinical Operations at Moffitt Cancer Center, and Jennie R. Crews, MD, MMM, Medical Director of the SCCA Network and Community Sites, were asked during the webinar whether their centers could return to their prepandemic telemedicine offerings, both responded with a resounding "no." Their response was attributed to the positive patient experience resulting from the convenience of telemedicine and the new demand to access it as an option in the future. Dr. Crews stated that SCCA is targeting to provide approximately $20 \%$ of oncology visits via telemedicine going forward compared with $5 \%$ prepandemic. This target is consistent with what several other NCCN Member Institutions have indicated through Best Practices Committee discussions, and also significantly higher than what the majority were providing before the pandemic. 


\section{Barriers to Telemedicine Will Need to be Overcome}

Although there are many benefits to telemedicine, there are also major challenges to address. As with any type of technology, systems used to conduct telemedicine visits are not always reliable. Providers and patients experience hardware, software, and internet connection issues that can be frustrating and difficult to address quickly. To mitigate technology problems, many centers have redesigned clinic workflows to include previsit technology checks, which increases staff burden and workload. Telemedicine also presents new equity concerns regarding patients' ability to access and use the technology.

Another disadvantage is the disconnectedness. A patient advocate who participated in the webinar series stated that although telemedicine visits are convenient and productive, they can fall short on the emotional support and personal connection many patients with cancer seek from their providers. Additionally, there remain unanswered questions about patient outcomes for telemedicine visits compared with in-person visits. Although initial feedback suggests that these visits have generally favorable outcomes, they are not a 1:1 substitute for face-to-face provider visits, and their future use is likely to be driven by specific-use cases rather than convenience alone.

Another major challenge faced by providers today is government regulations regarding the use of telemedicine. Dr. Crews noted that historically, barriers to telemedicine have included state and federal restrictions, low reimbursement, and difficulties obtaining provider credentialing to conduct telemedicine visits across state lines. As the benefits of telemedicine use during the pandemic became clear, the federal government temporarily waived geographic restrictions, relaxed HIPAA requirements, expanded provider eligibility, and increased reimbursement. ${ }^{10-12}$ These temporary changes in telemedicine regulations allowed for quick expansion of telemedicine offerings without fear of legal ramifications or incurring substantial financial repercussions.

Dr. Crews cautioned that some of these regulatory modifications are unlikely to remain in effect after the pandemic. This has caused concern for hospitals and cancer centers that have experienced the benefits of telemedicine and plan to continue with this effort. A recent proposal by the Centers for Medicare \& Medicaid Services (CMS) and emerging interest from Congress suggests there is a possibility that some modifications may remain. ${ }^{13,14}$ Additionally, a national taskforce developed by the National Committee for Quality Assurance (NCQA), the Alliance for Connected Care, and the American Telemedicine Association issued a report in mid-September of this year outlining telemedicine policy recommendations for this new era. The report advocates for keeping most of the coronavirus policy changes, including allowing telemedicine for various types of clinicians and conditions, and eliminating restrictions across state lines. ${ }^{15}$ The report makes general policy recommendations that are an important first step in ensuring appropriate patient access to telehealth services. As these services expand, it is critical that oncology professionals and other medical specialties be engaged to inform telehealth policy.

Dr. Kubal, who co-chairs the NCCN Best Practices Committee, provided advice for telemedicine expansion during his presentation for the webinar series. Although it is unclear at this point what the new postpandemic telemedicine regulations will entail, the increased demand for telemedicine will likely push centers to embrace this care delivery model to remain competitive and financially viable. Dr. Kubal suggests that cancer centers consider revising their cost structure (eg, decrease overhead/physical footprint) and revenue model (eg, reach more patients) to continue to realize at least $20 \%$ of visits (or more) via telemedicine. He suggests that centers focus on creating 2 distinct care models: (1) in-person visits focused on the patient experience, and (2) a telemedicine hybrid model based on patient convenience. He also recommended cancer centers offer a telemedicine tool that is easy to use for both patients and providers while delivering quality care that does not burden them or create new challenges. As per discussions from the NCCN Best Practices Committee, this will be challenging but necessary as cancer care moves forward.

\section{In-Person Visits Do Not Always Need to Take Place in a Hospital or Clinic}

The coronavirus pandemic has pushed cancer centers to find new ways to provide patient-centered, convenient care, even when telemedicine is not an option. When inperson care is required, some centers have developed creative ways to deliver this care to ensure safety and reduce the chances of viral spread. Similar to telemedicine, a high level of effectiveness and patient satisfaction with these new methods has centers planning for maintaining them postpandemic.

MiKaela Olsen, DNP, APRN, AOCNS, Clinical Nurse Specialist at The Sidney Kimmel Comprehensive Cancer Center at Johns Hopkins, discussed the development of curbside clinics during the pandemic. In an effort to reduce the number of people in the center and alleviate patients' fears regarding exposure, 2 curbside clinics were established for patients to receive treatment while in their vehicles. The curbside clinics offer chemotherapy injections (eg, bortezomib), blood draws, port flushes, immunizations, administration of growth factors, centralline flushing, and dressing changes for patients with cancer. In addition to patients appreciating that they could avoid 
entering the cancer center, they valued the convenience of not having to park and sit in a waiting room. Dr. Olsen stated that due to high patient satisfaction and demand, the Hopkins' curbside clinics will likely remain in effect after the pandemic. In fact, we may be seeing curbside clinics at other cancer centers, as well; Dr. Kubal indicated that Moffitt Cancer Center has plans to replicate Hopkins' curbside clinic model in the near future.

In February 2020, the Penn Center for Cancer Care Innovation at Abramson Cancer Center launched a pilot program to provide cancer treatment in the home setting, focusing on treatments being delivered in the infusion units or inpatient units. The program, called "Cancer Care at Home," rapidly expanded in March in response to coronavirus. Dr. Shulman stated that between mid-March and mid-June, oncologists' referrals for home administration of systemic therapy through the program increased by $1,000 \%$ (from 39 to 430 patients). The program, which began by providing leuprolide for patients with breast cancer, now provides 13 cancer drugs in the home setting for 5 diseases. Abramson Cancer Center is demonstrating that home cancer treatment can safely take the place of inpatient or outpatient administration for certain cancer drugs and patient populations. It has not only helped increase capacity but also saved patients significant time. Dr. Shulman estimates that a typical appointment to receive leuprolide at the center requires approximately 3 to 4 hours of committed patient time, whereas home treatment takes approximately 20 minutes. Due to the initial success of this endeavor, the center is taking steps to ensure that cancer treatment at home becomes a routine option going forward. Issues of reimbursement and patient copays are significant, though, and as with telemedicine, the profession should work with regulators and payers to adjust practice in ways that support optimal patient-centered care.

Cancer care experts agree that the pandemic has helped to eliminate low-value cancer care and shift delivery models to focus increasingly on patient convenience. ${ }^{16}$ Could it also provide a method for decreasing overall healthcare costs in the United States? When this question was posed during the Best Practices webinar series to Dr. Kubal and Dr. Crews, they both agreed that these creative strategies developed during the pandemic could assist in managing healthcare costs. The combination of expanding telemedicine and home care is likely to be the future of healthcare delivery and could play a significant role in decreasing overall costs. These exciting practice changes have the potential to become a standard lower-cost alternative, helping to reduce emergency department visits and hospitalizations.

\section{More Research Is Needed}

Although many cancer centers have already experienced the benefits of providing more patient-centered, convenient, and efficient care during the pandemic, either through telemedicine, curbside clinics, or in-home care, more time and research are needed to fully assess the impact and plan for the future. Dr. Shulman noted that Abramson Cancer Center will continue to collect outcomes data on telemedicine visits and home care to determine which patients are likely to benefit most from this type of care, as well as to ensure the safety of new approaches in different patient populations. This interest in outcomes as it relates to cancer care delivery modifications is also a priority at UCSF. Dr. Cinar noted that there is an increased focus on healthcare equity in regard to the innovative changes that have been implemented during the pandemic. How these new interventions may impact existing disparities in cancer care is an important area of consideration and collaboration for all cancer centers. These new ways of thinking and delivering care could amplify disparities in healthcare, but they could also serve as an opportunity to reduce or eliminate such disparities moving forward.

The immediate success associated with these alternative methods for providing cancer care is a strong indicator that they will become long-term wins in cancer care delivery when the pandemic has ended. We have an opportunity for patients, providers, payers, and regulators to come together to reassess our cancer care delivery system in the United States to the benefit of all, but this will take collective will. The hope is that we, as a country, will rise to the occasion.

Submitted August 28, 2020; accepted for publication September 28, 2020.

Disclosures: The authors have disclosed that they have not received any financial consideration from any person or organization to support the preparation, analysis, results, or discussion of this article. Ms. Sugalski is an employed by the National Comprehensive Cancer Network.

Correspondence: Jessica Sugalski, MPPA, National Comprehensive Cancer Network, 3025 Chemical Road, Suite 100, Plymouth Meeting, PA 19462. Email: sugalski@nccn.org

\section{References}

1. Institute of Medicine. Ensuring quality cancer care through the oncology workforce: sustaining care in the 21 st century: workshop summary. Washington, DC: National Academies Press; 2009.

2. Hortobagyi GN. A shortage of oncologists? The American Society of Clinical Oncology workforce study. J Clin Oncol 2007;25:1468-1469. https://doi.org/10.1200/JCO.2007.10.9397

3. Coughlin D. World Changing innovations that came out of global crises Accessed August 19, 2020. Available at: https://www.msn.com/en-za/ money/topstories/world-changing-innovations-that-came-out-of-globalcrises/ss-BB13BXaG?li=BBqfZdL\#image $=1$

4. Clark L. Innovation in a time of crises. Accessed August 19, 2020. Available at: https://www.harvardbusiness.org/innovation-in-a-time-of-crisis/ 
5. Institute of Medicine Committee on Evaluating Clinical Applications of Telemedicine. Field MJ, ed. Telemedicine: A Guide to Assessing Telecommunications in Health Care. Washington, DC: National Academies Press; 1996.

6. Board on Health Care Services; Institute of Medicine. The role of telehealth in an evolving health care environment: workshop summary. Washington, DC: The National Academies Press; 2012.

7. Bestsennyy O, Gilbert G, Harris A, Rost J. Telehealth: a quarter-trilliondollar post-COVID-19 reality? Accessed August 19, 2020. Available at: https://www.mckinsey.com/ /media/McKinsey/Industries/Healthcare\% 20Systems\%20and\%20Services/Our\%20Insights/Telehealth\%20A\% 20quarter\%20trillion\%20dollar\%20post\%20COVID\%2019\%20reality/ Telehealth-A-quarter-trilliondollar-post-COVID-19-reality.ashx

8. Powell RE, Henstenburg J, Cooper G, et al. Patient perceptions of telehealth primary care video visits. Ann Fam Med 2017;15:225-229.

9. Donelan K, Barreto EA, Sossong S, et al. Patient and clinician experiences with telehealth for patient follow-up care. Am J Manag Care 2019;25: 40-44.

10. U.S. Department of Health and Human Services. Notification of enforcement discretion for telehealth remote communications during the COVID-19 nationwide public health emergency. Accessed August 20, 2020. Available at: https://www.hhs.gov/hipaa/for-professionals/specialtopics/emergency-preparedness/notification-enforcement-discretion-telehealth/index.html

11. Federation of State Medical Boards. U.S. states and territories modifying requirements for telehealth in response to COVID-19. Accessed August
20, 2020. Available at: https://www.fsmb.org/siteassets/advocacy/pdf/ states-waiving-licensure-requirements-for-telehealth-in-response-tocovid-19.pdf

12. Centers for Medicare and Medicaid Services. COVID-19 emergency declaration blanket waivers for health care providers. Accessed August 20, 2020. Available at: https://www.cms.gov/files/document/summarycovid-19-emergency-declaration-waivers.pdf

13. Trump administration proposes to expand telehealth benefits permanently for Medicare beneficiaries beyond the COVID-19 public health emergency and advances access to care in rural areas [news release]. Baltimore, MD: Centers for Medicare and Medicaid Services; August 3 2020. Accessed August 19, 2020. Available at: https://www.cms.gov/ newsroom/press-releases/trump-administration-proposes-expand-telehealth-benefits-permanently-medicare-beneficiaries-beyond

14. Wicklund E. Congress aims to keep telehealth momentum going beyond the COVID-19 crisis. Accessed August 27, 2020. Available at: https:// mhealthintelligence.com/news/congress-aims-to-keep-telehealth-momentum-going-beyond-the-covid-19-crisis

15. Taskforce on Telehealth Policy (TTP) findings and recommendations. Latest evidence: September 2020. Accessed September 17, 2020. Available at: https://www.ncqa.org/wp-content/uploads/2020/09/ 20200914_Taskforce_on_Telehealth_Policy_Final_Report.pdf

16. Mullangi S, Schleicher SM, Aviki EM. Innovation in cancer care delivery in the era of COVID-19 [published online June 17, 2020]. JCO Oncol Pract, doi: 10.1200/OP.20.00336 